\title{
A Bayesian Partitioning Model for the Detection of Multilocus Effects in Case-Control Studies
}

\author{
Debashree Ray ${ }^{a} \quad$ Xiang Li ${ }^{a} \quad$ Wei Pan ${ }^{a}$ James S. Pankow ${ }^{b}$ Saonli Basu \\ Divisions of ${ }^{a}$ Biostatistics and ${ }^{b}$ Epidemiology and Community Health, School of Public Health, University of \\ Minnesota, Minneapolis, Minn., USA
}

\section{Key Words}

Dimension reduction - Multilocus interaction - Reversible jump Markov chain Monte Carlo

\begin{abstract}
Background: Genome-wide association studies (GWASs) have identified hundreds of genetic variants associated with complex diseases, but these variants appear to explain very little of the disease heritability. The typical single-locus association analysis in a GWAS fails to detect variants with small effect sizes and to capture higher-order interaction among these variants. Multilocus association analysis provides a powerful alternative by jointly modeling the variants within a gene or a pathway and by reducing the burden of multiple hypothesis testing in a GWAS. Methods: Here, we propose a powerful and flexible dimension reduction approach to model multilocus association. We use a Bayesian partitioning model which clusters SNPs according to their direction of association, models higher-order interactions using a flexible scoring scheme and uses posterior marginal probabilities to detect association between the SNP set and the disease. Results: We illustrate our method using extensive simulation studies and applying it to detect multilocus interaction in Atherosclerosis Risk in Communities (ARIC) GWAS with type 2 diabetes. Conclusion: We demonstrate
\end{abstract}

that our approach has better power to detect multilocus interactions than several existing approaches. When applied to the ARIC study dataset with 9,328 individuals to study gene-based associations for type 2 diabetes, our method identified some novel variants not detected by conventional single-locus association analyses.

(c) 2015 S. Karger AG, Basel

\section{Introduction}

The rapid progress in genotyping technology has greatly facilitated our understanding of the genetic predisposition to various diseases. Several genome-wide association studies (GWASs) have been published on various complex diseases, where genotype data on a large number of SNPs are collected to study the association between these SNPs and the disease. A common strategy to assess the effects of the SNPs on the disease is to perform a univariate regression with each SNP as a predictor and rank the SNPs based on their $\mathrm{p}$ values from the univariate regression analysis. The top significant SNPs that satisfy the genome-wide threshold of multiple testing are reported by the studies. Several such GWASs have successfully detected susceptibility SNPs associated with complex diseases such as type 2 diabetes [1], Crohn's disease and

\section{KARGER 125}

(c) 2015 S. Karger AG, Base

0001-5652/15/0792-0069\$39.50/0

E-Mail karger@karger.com

www.karger.com/hhe
Saonli Basu

Division of Biostatistics, School of Public Health

University of Minnesota

Minneapolis, MN 55455 (USA)

E-Mail saonli@umn.edu 
rheumatoid arthritis [2]. Due to huge computational requirements, most of these GWASs are often limited to single-SNP association analyses.

Multilocus association analyses such as gene-based associations have gained great impetus in recent years as the single-locus association findings have explained very little heritability of these complex traits. Moreover, with the advent of high-throughput sequencing technologies, there is a dire need to generate computationally efficient statistical methodologies to perform multilocus association analyses. Numerous recent studies [3-9] have developed multilocus association analysis techniques and software packages that evaluate the simultaneous association of multiple loci and traits. This large group of multilocus association analysis approaches can be classified into two broad categories: one that focuses on the detection of a subset of significant SNPs associated with a disease from a large group of loci (which include many null or notassociated loci) and the other that tests for association between a large set of loci and a disease without classifying each SNP to a null or non-null category [10-12].

The set of approaches that focus on the detection of a subset of significant SNPs from a large group of loci tends to concentrate on modeling only the main effects of the SNPs [3, 13-16]. There is evidence that diseases often arise as a result of complicated interactions among SNPs [17]. Hence, there could be a significant gain in power for the detection of associated loci by allowing higher-order interaction among these multiple SNPs. The major obstacle in modeling multilocus interaction is that the number of parameters increases exponentially with the number of loci. Thus, the approaches that allow for higherorder interaction need to incorporate variable selection or other dimension reduction techniques in their statistical model for associations between the SNP set and the disease [18-20]. Bayesian model selection or variable selection approaches offer an alternative technique for selecting multiple SNPs and interactions among them. Several Bayesian approaches have been developed that include efficient variable selection [21-24]. Fridley [25] has recently given an extensive overview on the Bayesian variable and model selection methods applied to genetic association studies. Lately, several attempts have been made to incorporate higher-order interaction in Bayesian multilocus modeling. Marttinen and Corander [26] used a model-searching algorithm starting from the marginal model to a saturated model to identify the optimal model for a combination of SNPs. Papathomas et al. [27] proposed a Bayesian nonparametric clustering approach combined with variable selection to search for gene-gene interaction. Another popular parametric approach to detect interaction under the Bayesian framework is the Bayesian Epistasis Association Mapping (BEAM) [23, 28, 29], which can handle large numbers of markers. This approach uses dimension reduction by classifying SNPs into 'null', 'main' or 'interaction' groups given their disease status. It still has limitations in terms of the number of loci that can be placed in the 'interaction' category, since the model uses the saturated model for the 'interaction' category.

This paper presents a new Bayesian methodology to detect multilocus effects incorporating the possibility of interaction among them in a case-control study setup. It aims to implement the data reduction strategy proposed by Basu et al. [30, 31] within a Bayesian framework by pooling the multilocus genotypes into 'low-risk', 'highrisk' and 'not-associated' categories based on the direction of effects and thus reducing the dimension of the genotype predictors from $p$ to 3 . An advantage over BEAM is that this approach can easily be extended to handle quantitative trait. Moreover, it does not use a saturated model for interaction but different scoring algorithms to capture higher-order interaction. We have considered two such scores to demonstrate the usefulness of the proposed model. Unlike Basu et al. [30, 31], our approach uses three parameters to classify the SNPs into 'low-risk', 'high-risk' and 'not-associated' categories and, hence, is expected to have better power to detect multilocus association. The not-associated SNPs are efficiently separated through Markov chain Monte Carlo (MCMC) updating, which also provides the posterior probability of each SNP in the SNP set being associated with the disease. In contrast to BEAM, our model does not distinguish between main effects or interaction effects of a group of SNPs, but our flexible scoring scheme captures high-order interaction effects successfully. Although our method can potentially be applied to scan a larger number of markers for association, it is more suitable to be used for a SNP set, such as for a gene or pathways, where associations are searched within each gene or pathway instead of the whole genome.

The Method section describes our Bayesian Partitioning Model (BPM) and the reversible jump MCMC (RJM$\mathrm{CMC}$ ) scheme in detail. Then, simulation results are presented to investigate the performance of a few existing methods and our BPM approach, demonstrating the advantages of the proposed method over several approaches. We conclude our Results section by illustrating the application of the methods to detect SNPs from a genebased association study with type 2 diabetes data on the 
Atherosclerosis Risk in Communities (ARIC) study. We finish with a short summary and discussion outlining a few future research topics.

\section{Method}

\section{A Dimension-Reducing Approach via BPM}

We propose a Bayesian approach to identify SNPs associated with a disease from a group of $p$ SNPs $(p \geq 2)$. The model employs the data reduction strategy proposed in Basu et al. [30, 31], models the joint effects of a group of SNPs on the trait and computes, via MCMC, the posterior probability of each SNP (or SNP set) of being associated with the disease. The dimension-reducing strategy is to assume that the minor allele of each SNP can be either of 3 types: (1) low risk (LR): the minor allele is associated with a decrease in the disease risk ('protective effect'); (2) not associated (NA): the minor allele has no effect on the disease, or (3) high risk (HR): the minor allele is associated with an increase in the disease risk ('deleterious effect').

Let $\mathbf{Y}=\left(y_{1}, \ldots, y_{n}\right)^{T}$ be the case-control status of $n$ unrelated individuals and $\mathbf{X}=\left(\mathbf{X}_{1}, \ldots, \mathbf{X}_{n}\right)^{T}$ be the $n \times p$ matrix of predictors. For the ease of explanation, we will assume that we only have data on SNPs. Hence $\mathbf{X}_{i}$ is a vector of the number of minor alleles of $p$ SNPs for the $i$-th individual. Each SNP can have 0,1 or 2 minor alleles. Let $\mathcal{A}_{j}$ denote the risk-label allocation of SNP $j ; j=1,2, \ldots, p$, where $\mathcal{A}_{j}=(0,1,0),(1,0,0)$ or $(0,0,1)$ denotes that SNP $j$ belongs to the $\mathrm{NA}, \mathrm{LR}$ or HR category, respectively. It should be noted that the choice of which allele is coded does not have an influence on our dimension-reducing strategy. It does not affect our conclusion because BPM detects SNPs associated with a disease. We do not know a priori if a SNP is NA, LR or HR. This is equivalent to the problem of model selection. For a set of $p$ SNPs, we consider the risk allocation matrix $\mathcal{A}$, where $\mathcal{A}=\left(\mathcal{A}_{1}, \ldots, \mathcal{A}_{p}\right)^{\prime}$ is a $p \times 3$ matrix. Hence, there are potentially $3^{p}$ choices of models, which we need to search in order to find the model that best explains the joint effect of the group of $p$ SNPs on the trait and compute the posterior probability of observing the best model (or risk allocation) given the trait and the marker data on the $n$ individuals.

Given a specific risk allocation $\mathcal{A}$, the effect of the group of $p$ SNPs is assessed using logistic regression:

$$
\log \left(\frac{P\left(y_{i}=1 \mid \boldsymbol{X}_{i}, \mathcal{A}\right)}{1-P\left(y_{i}=1 \mid \boldsymbol{X}_{i}, \mathcal{A}\right)} \mid \alpha, \beta_{1}, \beta_{2}\right)=\alpha+\beta_{1} Z_{1 i}+\beta_{2} Z_{2 i},
$$

where $\beta_{1}<0$ and $\beta_{2}>0$ define the fixed effects of the LR and the HR group of SNPs, and the predictors $Z_{1 i}$ and $Z_{2 i}$ are the values of scores for the LR and HR groups of an individual $i(i=1,2, \ldots, n)$, respectively. Note that the values of the predictors $Z_{1}$ and $Z_{2}$ depend on allocation $\mathcal{A}$. A particular choice of this score would be: $Z_{1 i}=$ the total number of minor alleles for the $i$-th individual in the LR group and $Z_{2 i}=$ the total number of minor alleles for the $i$-th individual in the HR group $(i=1,2, \ldots, n)$. We call it the 'M-score'. The flexibility of our method lies in the fact that many other scores can be proposed in order to capture the joint effect of the SNP set on the disease. We discuss another such choice of score in M-Score versus P-Score.

Next, we obtain the joint posterior distribution of $\mathcal{A}$ and $\beta$ as:

$$
P[\mathcal{A}, \beta \mid \mathrm{y}, \mathrm{X}] \propto P[\mathrm{y} \mid \mathbf{X}, \beta, \mathcal{A}] P[\beta \mid \mathcal{A}] P[\mathcal{A}] .
$$

Here, we use MCMC to study the joint posterior density given by equation 2. To construct the Markov chain, we make 3 simplifying assumptions in the model. First, we assume equal prior probabilities for a SNP to be in the 3 categories. If applied to a genome-wide dataset, a more informative choice of prior would be to assign much higher probability for each SNP to be in the NA (null) group [13], but we applied this model to the top genes identified by a gene-based association analysis. Hence, we decided to assign high probability for each SNP to be in the non-null group. Moreover, our choice of prior gave a simplified form (equation 4) of the acceptance probability in equation 3 . Second, we assume independent prior distributions of all SNPs, i.e.,

$$
P[\mathcal{A}]=\prod_{j=1}^{p} P\left[\mathcal{A}_{j}\right]=\text { constant } .
$$

Third, we assume $P[\beta \mid \mathcal{A}]=P[\beta]$, where $P[\beta]$ is the prior distribution of $\beta=\left(\alpha, \beta_{1}, \beta_{2}\right)$ following a truncated trivariate normal distribution: $\beta \sim N_{3}(\mu, V) \times I\left(\beta_{1}<0\right) \times I\left(\beta_{2}>0\right)$. We let the prior parameters $\mu$ be $(0,0,0)^{\prime}$ and set $V$ such that we expect $95 \%$ of the SNPs to have relative risks that lie within $\left[e^{-1.5}, e^{1.5}\right]$, as suggested by Wakefield et al. [24]. The diagonal of $V$ is, therefore, set at $\left\{1,0.207^{2}, 0.207^{2}\right\}$ and the off-diagonal elements are set to be 0 for an uncorrelated prior setting. The joint posterior distribution (equation 2) of $\mathcal{A}$ and $\beta$ lives on a high-dimensional product space. The SNP allocation label $\mathcal{A}$ lies on a discrete space $\{(0,1,0),(1,0,0)$, $(0,0,1)\}^{p}$, while $\beta \in \mathrm{R} \times \mathrm{R}^{-} \times \mathrm{R}^{+}$.

\section{Construction of the Markov Chain}

We construct a Markov chain using RJMCMC with 'dimension' moves, and 'allocation' and 'coefficient' moves within a fixed dimension. The dimension moves include 'death' and 'birth' steps to increase or decrease dimension $K$ by 1 . The dimension parameter $K$ can take 4 values: $0,1,2$, and 3, which refer to the case that the model has parameter(s) $\alpha ; \alpha$ and $\beta_{1} ; \alpha$ and $\beta_{2}$, and all three parameters $\alpha, \beta_{1}$ and $\beta_{2}$ in equation 1 , respectively. The first step in our RJMCMC is to choose one of the death, birth and fixed dimension moves at random. In a death step, we drop one parameter, randomly choosing between $\beta_{1}$ and $\beta_{2}$. In a birth step, we propose $\beta_{1}$ or $\beta_{2}$ and update $\mathcal{A}$ from its full conditionals (as described a little later). The acceptance probability for these dimension moves (from step $\overline{t-1}$ to step $\mathrm{t}$ ) is $\min \left(1, a\left(K^{(t-1)}, K^{(t)}\right)\right.$, with

$$
\begin{aligned}
& a\left(K^{(t-1)}, K^{(t)}\right)= \\
& \frac{P\left[K^{(t)}\right] P\left[\beta^{(t)}, \mathcal{A}^{(t)} \mid K^{(t)}\right] P\left[y \mid \mathcal{A}^{(t)}, \beta^{(t)}, K^{(t)}\right] P\left[K^{(t-1)} \mid K^{(t)}\right]}{P\left[K^{(t-1)}\right] P\left[\beta^{(t-1)}, \mathcal{A}^{(t-1)} \mid K^{(t-1)}\right] P\left[y \mid \mathcal{A}^{(t-1)}, \beta^{(t-1)}, K^{(t-1)}\right] P\left[K^{(t)} \mid K^{(t-1)}\right]} \\
& \times \frac{Q\left[D^{(t-1)} \mid D^{(t)}\right]}{Q\left[D^{(t)} \mid D^{(t-1)}\right]} \times|1|
\end{aligned}
$$

where $Q\left[D^{(t)} \mid D^{(t-1)}\right]$ is the proposal density of the move from model $D^{(t-1)}$ in step $\overline{t-1}$ to $D^{(t)}$ in step $t$. Since we assumed equal prior probabilities of a SNP to be in any of the 3 categories, $P$ $\left[K^{(t)}\right]=P\left[K^{(t-1)}\right]$. The 4 possible moves are random; hence, $P\left[K^{(t-1)}\right.$ $\left.\mid K^{(t)}\right]=P\left[K^{(t)} \mid K^{(t-1)}\right]$. Also, $P\left[\beta^{(t)}, \mathcal{A}^{(t)} \mid K^{(t)}\right]=P\left[\beta^{(t)} \mid \mathcal{A}^{(t)}, K^{(t)}\right] P$ $\left[\mathcal{A}^{(t)} \mid K^{(t)}\right]$. The possible moves along with the corresponding acceptance probabilities are listed in online supplementary material section 1 (see www.karger.com/doi/10.1159/000369858 for all online suppl. material). 
We now look into the general form of the proposal density $Q$ $\left[D^{(t)} \mid D^{(t-1)}\right]$. Note that:

$$
\begin{aligned}
Q\left[D^{(t)} \mid D^{(t-1)}\right] & =P\left[\beta^{(t)}, \mathcal{A}^{(t)}, K^{(t)} \mid \beta^{(t-1)}, \mathcal{A}^{(t-1)}, K^{(t-1)}\right] \\
& =P\left[\beta^{(t)} \mid \mathcal{A}^{(t)}, K^{(t)}, \beta^{(t-1)}, \mathcal{A}^{(t-1)}, K^{(t-1)}\right] \\
& \times P\left[\mathcal{A}^{(t)} \mid K^{(t)}, \beta^{(t-1)}, \mathcal{A}^{(t-1)}, K^{(t-1)}\right] \\
& \times P\left[K^{(t)} \mid \beta^{(t-1)}, \mathcal{A}^{(t-1)}, K^{(t-1)}\right] \\
& =P\left[\beta^{(t)} \mid \mathcal{A}^{(t)}, K^{(t)}\right] P\left[\mathcal{A}^{(t)} \mid K^{(t)}\right] P\left[K^{(t)} \mid K^{(t-1)}\right] .
\end{aligned}
$$

So, equation 3 reduces to the simple form of a likelihood ratio:

$$
a\left(K^{(t-1)}, K^{(t)}\right)=\frac{P\left[y \mid \beta^{(t)}, \mathcal{A}^{(t)}, K^{(t)}\right]}{P\left[y \mid \beta^{(t-1)}, \mathcal{A}^{(t-1)}, K^{(t-1)}\right]} .
$$

We obtain $P\left[\mathrm{y} \mid \beta^{(t)}, \mathcal{A}^{(t)}, K^{(t)}\right]$ and $P\left[\mathrm{y} \mid \beta^{(t-1)}, \mathcal{A}^{(t-1)}, K^{(t-1)}\right]$ using the model in equation 1 . Within a fixed dimension, we update the Markov chain through allocation and coefficients moves, i.e., we first update $\mathcal{A}$ from its full conditionals and then update $\beta$ using a Metropolis-Hastings algorithm.

\section{Updating $\mathcal{A}_{j}$ from Full Conditionals}

We assume a multinomial prior for the configuration of SNP $\mathcal{A}_{j}$ and equal prior probabilities of being in the LR, NA, and HR categories. So, $\mathcal{A}_{j} \sim$ Multinomial $\left(m=1 ; p_{j 1}=1 / 3, p_{j 2}=1 / 3, p_{j 3}=\right.$ $1 / 3)$, where $\mathcal{A}_{j} \in\left\{(1,0,0)^{\prime},(0,1,0)^{\prime},(0,0,1)^{\prime}\right\}$. If $\mathcal{A}_{(-j)}$ denotes configuration of all SNPs except the $j$-th SNP, then the full conditional of $\mathcal{A}_{j}$ at step $t$ also has a multinomial distribution:

$$
\left[\mathcal{A}_{j}^{(t)} \mid \beta^{(t-1)}, \mathcal{A}_{(-j)}^{(t-1)}\right] \sim \text { Multinomial }\left(m=1 ; p_{j 1}^{(t)}, p_{j 2}^{(t)}, p_{j 3}^{(t)}\right),
$$

where $p_{j 1}^{(t)}, p_{j 2}^{(t)}$ and $p_{j 3}^{(t)}$ are the posterior probabilities of SNP $j$ to be in the LR, NA and HR group, respectively. These posterior probabilities are given by:

$$
p_{j s}^{(t)}=\frac{P\left[\boldsymbol{y} \mid \beta^{(t-1)}, \mathcal{A}_{(-j)}^{(t-1)}, \mathcal{A}_{j}^{(t)}=\boldsymbol{a}_{s}\right] P\left[\mathcal{A}_{j}^{(t)}=\boldsymbol{a}_{s}\right]}{\sum_{k=1}^{3} P\left[\boldsymbol{y} \mid \beta^{(t-1)}, \mathcal{A}_{(-j)}^{(t-1)}, \mathcal{A}_{j}^{(t)}=\boldsymbol{a}_{k}\right] P\left[\mathcal{A}_{j}^{(t)}=\boldsymbol{a}_{k}\right]},
$$

where $s=1,2,3, \mathrm{a}_{s} \in\left\{(1,0,0)^{\prime},(0,1,0)^{\prime},(0,0,1)^{\prime}\right\}$ and

$$
P\left[\boldsymbol{y} \mid \beta^{(t-1)}, \mathcal{A}_{(-j)}^{(t-1)} \mathcal{A}_{j}^{(t)}=\boldsymbol{a}_{s}\right]=P\left[\boldsymbol{y} \mid \beta^{(t-1)}, \mathcal{A}_{(-j)}^{(t-1)} \mathcal{A}_{j}^{(t)}=\boldsymbol{a}_{s}, K^{(t)}\right]
$$

is obtained using the model in equation 1.

Updating $\beta$ Using a Metropolis-Hastings Algorithm

After updating $\mathcal{A}$ from its full conditionals and getting $\mathcal{A}^{(t)}$, we sample $\beta^{*}$ from the proposal density $N_{3}\left(\beta^{(t-1)}, \mathbf{V}\right) I\left(\beta_{1}<0\right) I\left(\beta_{2}>\right.$ $0)$. For each draw of $\beta^{*}$ from the proposal, we accept $\beta^{*}$ as $\beta^{(t)}$ with probability $\min \left(1, a^{\prime}\left(\beta^{(t-1)}, \beta^{*}\right)\right)$, where

$$
a^{\prime}\left(\beta^{(t-1)}, \beta^{*}\right)=\frac{P\left[\boldsymbol{y} \mid \beta^{*}, \mathcal{A}^{(t)}\right] \cdot P\left[\beta^{*} \mid \mathcal{A}^{(t)}\right]}{P\left[\boldsymbol{y} \mid \beta^{(t-1)}, \mathcal{A}^{(t)}\right] \cdot P\left[\beta^{(t-1)} \mid \mathcal{A}^{(t)}\right]} \times \frac{P\left[\beta^{(t-1)} \mid \beta^{*}\right]}{P\left[\beta^{*} \mid \beta^{(t-1)}\right]} .
$$

Note that $P\left[y \mid \beta^{*}, \mathcal{A}^{(t)}\right]=P\left[y \mid \beta^{*}, \mathcal{A}^{(t)}, K^{(t)}\right]$ and $P\left[y \mid \beta^{(t-1)}, \mathcal{A}^{(t)}\right]$ are obtained from the model in equation 1 . The implementation of this RJMCMC is outlined in detail in online supplementary material section 2 .

\section{M-score versus P-score}

The M-score corresponds to a model (equation 1), where $Z_{1}$ and $Z_{2}$ are the total number of minor alleles in the LR and HR group, respectively. The $\mathrm{M}$-score technique is theoretically equivalent to considering only the main effects of SNPs in a logistic regression model with equal effect sizes of the SNPs in the LR group and equal effect sizes of the ones in the HR group. For example, let us consider allocation $\mathcal{A}$, where the first $p_{1}$ SNPs are in the LR group and the remaining $p_{2}$ SNPs are in the HR group, $p_{1}+p_{2}=p$. Thus, for individual $i$, the M-score for the LR group is

$$
Z_{1 i}=\sum_{k=1}^{p_{1}} X_{i k}
$$

and that for the HR group is

$$
Z_{2 i}=\sum_{j=1}^{p_{2}} X_{i j}
$$

Equation 1 becomes

$$
\begin{aligned}
& \quad \operatorname{logit}\left(P\left[y_{i}=1 \mid \mathbf{X}_{i}, \beta, \mathcal{A}\right]\right)=\alpha+\beta_{1} X_{i 1}+\ldots+\beta_{1} X_{i p_{1}}+\beta_{2} X_{i\left(p_{1}+1\right)}+\ldots \\
& +\beta_{2} X_{i p .}
\end{aligned}
$$

Now we propose a pairwise score to capture higher-order interaction among the SNPs. The P-score is calculated as the total number of pairs of minor alleles in the LR and HR groups. To implement the $\mathrm{P}$-score in equation 1 , we define $Z_{1 i}$ as the number of unordered samples of minor alleles of size 2 (without replacement) from the total number of minor alleles in the LR group of individual $i(i=1$, $2, \ldots, n)$. Similarly $Z_{2 i}$ is defined for the HR group. $Z_{1 i}=1$ and $Z_{2 i}=$ 1 , when there are only 2 minor alleles in the LR and HR group of the $i$-th individual, respectively. A score of 0.5 is arbitrarily assigned, if there is only 1 minor allele in a group.

Each allocation $\mathcal{A}$ and the corresponding P-score is equivalent to a multiple logistic regression model with predictors as some function of the main effects and the pairwise interaction among the SNPs. For our hypothetical example with the first $p_{1}$ SNPs in the LR group and the remaining $p_{2}$ SNPs in the HR group, we consider the multiple logistic regression model in equation 1 :

$$
\begin{aligned}
& \operatorname{logit}\left(P\left[y_{i}=1 \mid \boldsymbol{X}_{i}, \beta, \mathcal{A}\right]\right) \\
& =\alpha+\beta_{1}\left(\begin{array}{c}
\sum_{k=1}^{p_{1}} X_{i k} \\
2
\end{array}\right)+\left(\begin{array}{c}
\sum_{j=1}^{p_{2}} X_{i j} \\
2
\end{array}\right) \\
& =\alpha+\beta_{1}\left(\sum_{s, t: s<t} X_{i s} X_{i t}+\sum_{k=1}^{p_{1}} X_{i k}\left(X_{i k}-1\right) / 2\right) \\
& +\beta_{2}\left(\sum_{l, m: l<m} X_{i l} X_{i m}+\sum_{j=1}^{p_{2}} X_{i j}\left(X_{i j}-1\right) / 2\right) .
\end{aligned}
$$

Thus, through the P-score we can theoretically capture the main effects as well as the pairwise interaction effects among the SNPs. In practice, our simulation studies show that the P-score can capture higher-order interaction effects as well (see Simulation 1). Our simulation study (refer to online suppl. material section 3 ) using 1,000 cases and 1,000 controls shows the advantage of the proposed pairwise score modeling (P-score) over the main effect modeling (M-score) in the presence of interaction. One can use other scoring schemes, such as Gaussian kernels, to capture interaction among SNPs. 


\section{Results}

We performed several simulation studies to demonstrate the importance of the choice of scores for our model and to compare our approach with some existing ones.

\section{Simulation 1}

We first compared our BPM approach with BEAM [23] using simulation studies on uncorrelated SNPs. We also compared our approach with the logistic kernel machine (LKM) regression method. The kernel machine regression tests [10] are computationally efficient tests which score similarity among individuals through different choices of kernels, such as linear (LKM-linear), identity-by-descent (LKM-ibs), quadratic (LKM-quadratic), and use a score test to detect association between the SNP set and the disease status.

We simulated data on 20 uncorrelated SNPs with 200 cases and 200 controls. Only the first 4 SNPs were associated with the case-control status. We considered 5 epistatic models with different main effect sizes (and directions) and interaction effect sizes. Two-way, three-way and four-way interactions were considered. We considered both additive and dominant genetic models for this power comparison. The following models were used in our simulations:

Model 1: $\operatorname{logit}(\mathrm{p})=-4+1 / 5 X_{1}+1 / 5 X_{2}-X_{3}-X_{4}$, where $X_{j}=0$, 1,2 denote SNP $j$ with $0,1,2$ minor alleles, respectively.

Model 2: $\operatorname{logit}(\mathrm{p})=-4-2 X_{4}+X_{1} X_{2} X_{3}$, where $X_{j}=0,1,2$ denote SNP $j$ with $0,1,2$ minor alleles, respectively.

Model 3: $\operatorname{logit}(\mathrm{p})=-4+2 X_{1} X_{2} X_{3} X_{4}$, where $X_{j}=0,1,2$ denote SNP $j$ with $0,1,2$ minor alleles, respectively.

Model 4: $\operatorname{logit}(\mathrm{p})=-4+1 / 2 X_{1} X_{2}+X_{1} X_{3}+X_{3} X_{4}$, where $X_{j}=0$, 1 for SNP $j$ with $0, \geq 1$ minor alleles, respectively.

Model 5: $\operatorname{logit}(\mathrm{p})=-4+1 / 5 X_{1}+2 / 5 X_{2}+3 / 5 X_{3}-X_{4}$, where $X_{j}=0,1,2$ denote SNP $j$ with 0, 1, 2 minor alleles, respectively.

For each of these models, we simulated 200 datasets with each SNP at a minor allele frequency (MAF) of 0.2. Using ROCs, we first compared the power of our BPM Mscore and P-score approaches with that of BEAM to detect genetic variants associated with the disease. Here, we are interested in testing the null hypothesis that a chosen SNP is null. For every simulated model, we considered a range of cutoffs between 0 and 1, and for each cutoff, we calculated the number of times the posterior probabilities of each of the associated SNPs, such as SNP1, SNP2, SNP3, and SNP4, in the non-null category was higher than the cutoff value. We also calculated the number of times the posterior probabilities of each of the truly null SNPs was higher than the cutoff out of 200 simulations. We generated a ROC curve by calculating the average number of truly associated SNPs (true positive rate) detected and the
Table 1. Comparison of BPM and BEAM

\begin{tabular}{llllll}
\hline Method & \multicolumn{5}{l}{ Simulated Model } \\
\cline { 2 - 6 } & 1 & 2 & 3 & 4 & 5 \\
\hline BPM M-score & 0.48 & 0.29 & 0.15 & 0.23 & 0.22 \\
BPM P-score & 0.41 & 0.31 & 0.55 & 0.21 & 0.19 \\
BEAM & 0.26 & 0.30 & 0.25 & 0.05 & 0.15 \\
\hline
\end{tabular}

Power of the 3 methods in detecting the 4 associated SNPs for Bonferronis corrected error level of $0.0025(=0.05 / 20)$ based on 200 datasets with 200 cases and 200 controls.

average number of false positives (false positive rate) detected by BPM and BEAM for a given cutoff. The average number of false positives detected by BPM gives an estimate of the type I error in BPM when testing if a chosen SNP is null. For BPM, we ran a single chain of size 10,000. The first 5,000 were discarded as burn-in. For BEAM, we took the default chain size of 100,000 with a burn-in of 50,000 . The thin parameter was set at 1 . The default prior probabilities of 0.01 were used for each SNP to belong to the marginal or interaction groups.

According to figure 1 and table 1, our BPM approach outperformed BEAM for all 5 models at a Bonferronicorrected level of $0.0025(=0.05 / 20)$ except for Model 2, where the performances were very similar. For Model 1, we only had main effects under an additive genetic model. BEAM had a lower true positive rate than BPM for a false positive rate $<0.1$. Especially the $\mathrm{M}$-score performed well due to its ability to capture the main effects successfully. For a Bonferroni corrected level of $0.0025, \mathrm{BPM}$ had a power of 0.48 , while BEAM had only 0.26 .

For Model 2, as soon as we added an interaction term to a main effect model, the P-score of BPM had uniformly better power than both the M-score and the BEAM due to its ability to capture interaction effects. Even in the presence of a strong main effect, the M-score could not outperform the P-score. Here, BEAM performed marginally better than the M-score of BPM.

The same was true for Model 3 (an interaction-only model), where BEAM outperformed the M-score marginally, but the P-score captured the four-way interaction efficiently and outperformed BEAM. Here, while BEAM had a very low true positive rate of 0.25 at a false positive rate of 0.0025 , the P-score had a good true positive rate of 0.55 (table 1). To see if BPM really performs better than BEAM in capturing higher-order interactions, we also considered another additive genetic model (no figure shown), where the first 5 out of 20 independent SNPs 

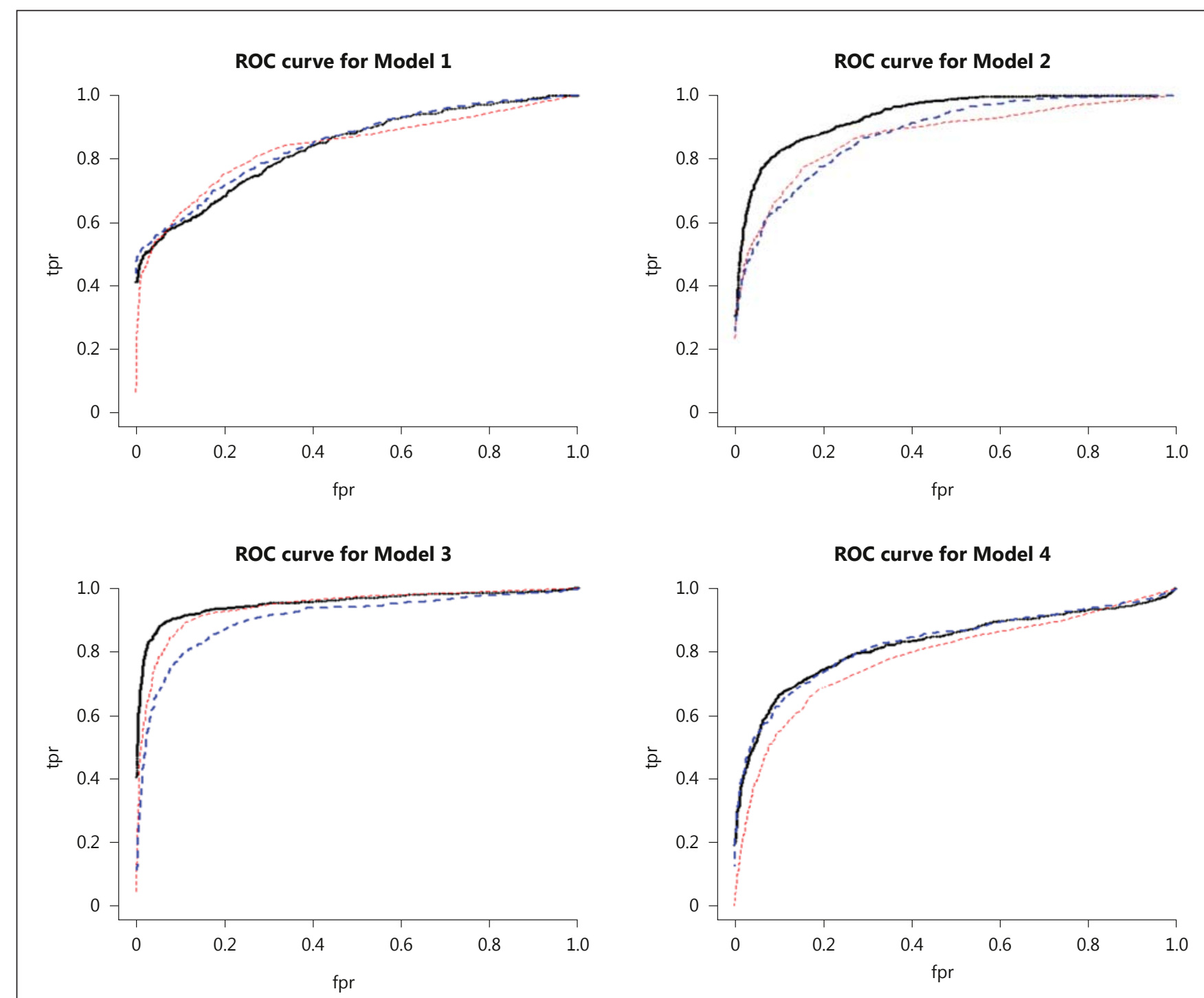

ROC curve for Model 5

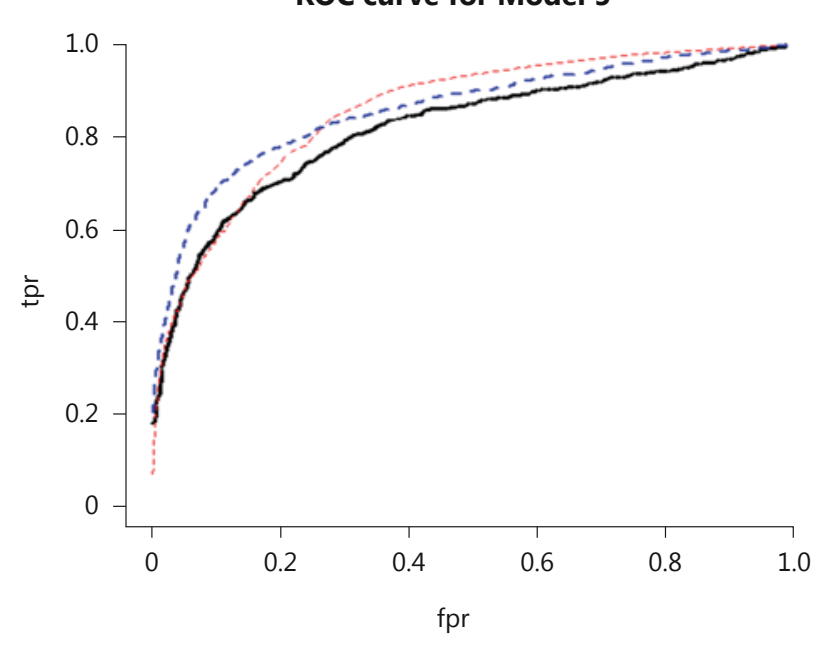

- BPM P-score

- BPM M-score

-.. BEAM

1

(For legend see next page.) 
Table 2. Comparison of BPM, BEAM and LKM

\begin{tabular}{lllll}
\hline Method & \multicolumn{5}{l}{ Simulated Model } & \\
\cline { 2 - 5 } & 1 & 2 & 3 & 4 \\
\hline BPM M-score & 1.00 & 1.00 & 0.41 & 0.50 \\
BPM P-score & 0.93 & 0.98 & 0.91 & 0.48 \\
BEAM & 0.81 & 1.00 & 0.34 & 0.12 \\
LKM-linear & 0.91 & 0.995 & 0.84 & 0.56 \\
LKM-quadratic & 0.89 & 1.00 & 0.96 & 0.64 \\
LKM-ibs & 0.93 & 0.99 & 0.80 & 0.69 \\
\hline
\end{tabular}

Power of the 6 methods in detecting at least 1 of the 4 associated SNPs for Bonferroni's corrected error level of $0.0025(=0.05 / 20)$ based on 200 datasets with 200 cases and 200 controls.

were causal and were interacting with each other to increase the disease risk. The P-score of BPM had uniformly better power than BEAM again.

For Model 4, we considered a dominant genetic model with only pairwise interaction effects. The true effect sizes being small, all of the methods lost some power under the dominant model, but BPM had better power than BEAM (except for error levels close to 1). The M-and Pscores of BPM performed similar according to the ROC curve (fig. 1).

For Model 5, our method outperformed BEAM even when the basic model assumption of equal effect size was violated for our BPM approach. BEAM had lower power than BPM for an error level of $<0.2$, especially for the $\mathrm{M}$ score due to its ability to capture the main effects successfully.

Next, we compared the powers of BPM, BEAM and LKM methods to detect multilocus association. Our null hypothesis of interest is that none of the SNPs is associated with the disease status. Since LKM regression can only test if a group of SNPs is associated or not at a given type I error level, we calculated the number of times each of the BEAM and BPM approaches detected at least one causal SNP out of the 4 SNPs for varying error levels. Table 2 (and online suppl. material fig. 2) shows that at a Bonferroni adjusted error of 0.0025 , BPM had better

Fig. 1. The ROCs for BPM (the M- and P-scores) and BEAM for the 5 epistatic models with 4 causal SNPs. The true positive rate (tpr) or sensitivity for each dataset was calculated as the proportion of causal SNPs detected based on their posterior marginal probabilities in the non-null category and for a series of cutoffs for the posterior probabilities. (The ratio of positive SNPs to non-null SNPs.) It was averaged across the 200 simulated datasets with 20 power over BEAM and LKM regression for the main-effect-only model (Model 1). All three methods had comparable power when there was a main effect and an interaction effect in the model (Model 2). In the presence of only a fourth-order interaction (Model 3) or several pairwise interaction effects (Model 4), LKM regression had the best performance closely followed by the BPM approach. One thing to keep in mind is that LKM regression can only test for association between a SNP set and a disease; its limitation is that it cannot specifically identify the null and the non-null SNPs.

\section{Simulation 2}

Here, we performed a simulation study with correlated SNPs to see the impact of linkage disequilibrium (LD) on our BPM approach. We considered correlation coefficients of $\rho=0,0.5$ and 0.9 . The SNPs were simulated from a latent multivariate Gaussian variable with an $\operatorname{AR} 1(\rho)$ structure. As before, we simulated 200 datasets on 200 cases and 200 controls with 20 SNPs (the first 4 are causal) at MAF 0.2. We performed this comparison with the Mscore and with Model 1 . The power of BPM for the 3 different correlations $(\rho=0,0.5,0.9)$ at various error levels were plotted (see online suppl. material section 5). At low type I error levels, BPM lost power with the increase in LD values among the SNPs. For higher error levels, the power of the BPM M-score approach to detect association was similar for $\rho=0$ and $\rho=0.5$. This observation was not consistent for all of our simulations. We noticed sometimes gain in power for $\rho=0.5$ over $\rho=0$, but fall in power for high correlations like $\rho=0.9$, especially for models with interaction effect. In summary, a moderate correlation among SNPs did not affect the performance of the BPM approach significantly but for high SNP-SNP correlations, $\mathrm{BPM}$ lost power at stringent error levels.

\section{Convergence Diagnostics}

Checking the convergence of RJMCMC is not straightforward. The general consensus is to monitor common parameters (in our case, $\alpha$ ) using popular fixed-dimensional convergence diagnostics [32]. Gelman and Rubin's diagnostic [33] gave a point estimate for the median potential calculated. (The ratio of negative SNPs to null SNPs.) As the cutoff for the posterior probability was varied, the fpr also varied. An increasing order of fpr is plotted along the $\mathrm{x}$-axis and of tpr along the $\mathrm{y}$-axis. Here, the heavy black curve represents the P-score of BPM, the heavy blue dashed curve is the M-score of BPM and the light red dashed curve is BEAM (colors refer to the online version only). 
scale reduction factor for $\alpha$ as $1.00(<1.1$ means the chain has converged to the stationary distribution and we need not run the chain longer). We also plotted posterior distributions of all 3 parameters $\alpha, \beta_{1}$ and $\beta_{2}$ for 6 independent chains (using the $\mathrm{M}$-score) for a randomly chosen dataset under Model 1 (main-effect-only model) with uncorrelated SNPs (online suppl. material section 6). The starting parameters $\beta$ and $\mathcal{A}$ were different for each chain. Convergence was achieved for all these chains. The mean values of $\beta_{1}$ and $\beta_{2}$ (averaged over all 6 chains) were respectively $-0.96(\mathrm{SD}=0.18)$ and $0.23(\mathrm{SD}=0.15)$, which were close to the true effect sizes of -1 and 0.2 , respectively.

In online supplementary material figure 4 , we presented summaries of $\beta_{1}$ and $\beta_{2}$ (using boxplots) for 15 randomly chosen datasets out of 200 datasets simulated under Model 1. In this model, the effect size of SNPs with negative direction (protective or LR SNP) was -1 and that of SNPs with positive direction (deleterious or HR SNP) was 0.2. Since we are looking at M-score results, we expect the estimated $\beta_{1}$ and $\beta_{2}$ to be close to -1 and 0.2 , respectively. Online supplementary material figure 4 shows that the estimates align quite well with the true values.

We also looked at the convergence of multivariate categorical parameter $\mathcal{A}$. For this purpose, we randomly selected 3 SNPs (out of 20), each of which was known to belong to the 3 different groups LR, NA and HR (Model 1). Online supplementary material figure 6 graphically compares the posterior probabilities of each of the chosen SNPs to belong to each of the groups across 6 independent chains. Stability of the posterior probabilities of the various categories over independent chains indicates convergence.

\section{Real Data Analysis}

Extensive evidence, including that gathered from twin and family studies, supports the hypothesis that genetic factors are a major contributor to the risk of type 2 diabetes (T2D). More recently, a GWAS of T2D conducted in populations of European ancestry has identified more than 50 SNPs reaching genome-wide levels of significance, most of which appear to act in the pancreatic betacell development or function [1,34]. Several GWASs of related quantitative traits such as fasting glucose have offered additional signals. These loci are significant contributors to the risk of T2D with population-attributable risks $>5 \%$ per locus in many cases. These results provide strong evidence for the existence and identification of common genetic risk factors for T2D.

The ARIC study is an ongoing prospective study designed to investigate the etiology and natural history of atherosclerosis and its clinical manifestations, and to mea- sure variation in cardiovascular risk factors, medical care and disease by race, gender, place and time [35]. ARIC has collected fasting glucose measures from the entire cohort at 4 separate visits over a 9 -year period and self-reported physician diagnosis and medication use in up to 14 separate interviews over a 20 -year period. Diabetes was defined as fasting glucose $\geq 126 \mathrm{mg} / \mathrm{dl}$, nonfasting glucose $\geq 200 \mathrm{mg} / \mathrm{dl}$, self-reported physician diagnosis of diabetes or current use of diabetes medications. Details about the ARIC study samples and their genotyping can be found in online supplementary material section 10 .

We conducted single-SNP association analyses on the Caucasian subset (sample size $=9,328$ with 812 cases) using PLINK [36] and a gene-based association analysis in VEGAS [37]. As per VEGAS, the two strongest signals were located in genes TCF7L2 (gene p value $=8 \times 10^{-6}$ ) on chromosome 10 and MMRN1 (gene p value $=5.7 \times 10^{-5}$ ) on chromosome 4. PLINK identified the SNPs rs7903146 (p value $\left.=1.7 \times 10^{-11}\right)$ and $\mathrm{rs} 1318557\left(\mathrm{p}\right.$ value $\left.=2.5 \times 10^{-6}\right)$ to be the most significant SNPs of genes TCF7L2 and MMRN1, respectively. Since VEGAS gives only gene-based $\mathrm{p}$ values, we wanted to explore if some additional SNPs in these two genes could be identified by our BPM approach, which were not detected in the single-SNP association analysis.

To implement our BPM approach, we again focused on the Caucasian participants. We implemented the BPM approach on the SNP data separately for each of the two genes mentioned above. We followed the same definition used by VEGAS for the allocation of SNPs to the genes. Our goal was to analyze each of the genes separately using our BPM approach (both the M- and P-scores), find the optimal allocation of the SNPs within each gene and compare the performance of the BPM with single-SNP association findings.

For the gene-based association analysis, we excluded all SNPs with MAF $<5 \%$ and all SNPs with an absolute pairwise correlation coefficient $|\rho|>0.8$ with another SNP. For a given gene data, we computed posterior probabilities for the minor allele of each of the SNPs to be in each of the 3 categories (LR, NA and HR) based on a long chain of 500,000 MCMC iterations. Within each iteration, $\beta$ is iterated 10 times. The posterior probability of a SNP to be in a particular group was calculated as the average number of times that SNP was allocated in that group in each MCMC iteration. The starting allocation was randomly generated for each chain. Using HeidelbergerWelch tests [38], the burn-ins were decided for each gene and each score to ensure stationarity of the common parameter $\alpha$ of each chain at a $5 \%$ significance level. 
Given the posterior probabilities of a SNP in the LR, NA and HR group, we used a cutoff of 0.4 for the non-NA $(\mathrm{LR}+\mathrm{HR})$ posterior probability to assign a SNP into a non-NA group. Any SNP with a non-NA (LR+HR) posterior probability exceeding the threshold was assigned to be non-NA. The allocation of a non-NA SNP to the LR or HR group was based on which group had the higher posterior probability among the two. For each score and each gene, we thus obtained the final allocation $\mathcal{A}$ of the SNPs and calculated the approximate Bayes Factor (ABF) [39] as a measure of evidence in favor of the null or the alternative hypothesis of association.

For the calculation of $\mathrm{ABF}_{01}$ (the posterior odds of null model to the alternative model $\mathcal{A}$, selected using $\geq 0.4$ posterior probability for a SNP to belong to a non-NA group), we evaluated the joint likelihood of $\boldsymbol{Y}$ and $\boldsymbol{\beta}$ under the null as well as under the alternative hypothesis. Since there is no closed form of the joint likelihood, we used the Laplace approximation around the maximum a posteriori (MAP) estimate of $\boldsymbol{\beta}$ to obtain the null likelihood. On the other hand, we computed the alternative joint likelihood by using the Laplace approximation around the posterior mode of $\boldsymbol{\beta}$ obtained from the posterior samples [40]. In our calculations, we found the posterior mode to be almost the same as the MAP estimate of $\beta$ under the alternative hypothesis.

We first analyzed gene MMRN1 on chromosome 4 with 57 SNPs after screening. For BPM, the M-score chain for $\alpha$ passed the Heidelberger-Welch stationarity test and the half-width mean test at the $5 \%$ level without any burn-in, while a burn-in of 100,000 was needed for the P-score. Online supplementary material figure 5 also shows the convergence of these two chains. At a cutoff of 0.4, only 4 SNPs were detected as LR for the M-score, which included the most significant SNP rs1318557 ( $\mathrm{p}$ value $=2.5 \times 10^{-6}$ ) from the single-SNP association analysis (table 3). The posterior probability of this SNP to belong to the LR category was 0.47 . The other 3 SNPs chosen by BPM were not at all significant in the single-SNP association analysis (table 3 ). The $\mathrm{M}$-score had $-2 \log _{10}$ $\left(A B F_{01}\right)=10.5>10$, which indicated very strong evidence of association [40].

On the other hand, the P-score could not detect any SNP as LR/HR at the chosen cutoff, although at a lower cutoff of 0.2 , it detected these above-mentioned SNPs. This may indicate the fact that these SNPs in MMRN1 are not contributing through interactions, and hence the Pscore could not perform as well as the M-score.

We next analyzed gene TCF7L2 on chromosome 10 with 109 SNPs after screening. Using Heidelberger-Welch
Table 3. The final allocation of the non-null SNPs selected by using a cutoff of 0.4 on the posterior probability of each SNP to be in the non-NA (LR+HR) group from a BPM M-score analysis in the MMRN1 gene

\begin{tabular}{lllll}
\hline & \multirow{2}{*}{$\begin{array}{l}\text { Posterior } \\
\end{array}$} & $\mathcal{A}$ & $\begin{array}{l}\text { Posterior } \\
\text { probability }\end{array}$ & \multicolumn{2}{c}{ Single-SNP } & \\
\cline { 4 - 5 } & & coefficients & p values \\
\hline rs11727074 & LR & 0.38 & -0.2 & $6.2 \times 10^{-2}$ \\
rs6812192 & LR & 0.48 & -0.2 & $1.4 \times 10^{-3}$ \\
rs12646270 & LR & 0.43 & -0.3 & $4.0 \times 10^{-2}$ \\
rs1318557 & LR & 0.47 & -0.3 & $2.5 \times 10^{-6}$ \\
\hline
\end{tabular}

The single-SNP results of these non-null SNPs are also listed for comparison. The direction of the single-SNP coefficient and the group allocation by BPM match.

Table 4. The final allocation of the non-null SNPs selected by using a cutoff of 0.4 on the posterior probability of each SNP to be in the non-NA (LR+HR) group from a BPM M-score analysis in the TCF7L2 gene

\begin{tabular}{lllll}
\hline & \multirow{2}{*}{$\begin{array}{l}\text { Posterior } \\
\end{array}$} & $\mathcal{A}$ & $\begin{array}{l}\text { Posterior } \\
\text { probability }\end{array}$ & \multicolumn{2}{c}{ Single-SNP } & \\
\cline { 5 - 5 } & & coefficients & p values \\
\hline rs17747324 & HR & 0.46 & 0.42 & $3.6 \times 10^{-11}$ \\
rs7903146 & LR & 0.48 & -0.38 & $1.7 \times 10^{-11}$ \\
\hline
\end{tabular}

The single-SNP results of these non-null SNPs are also listed for comparison. The direction of the single-SNP coefficient and the group allocation by BPM match.

tests at the 5\% level, burn-ins of 200,000 and 150,000 for the $\mathrm{M}$ - and P-scores, respectively, ensured stationarity for common parameter $\alpha$. At a cutoff of 0.4 , with $-2 \log _{10}$ $\left(A B F_{01}\right)=15.7>10$, the M-score found only the 2 SNPs rs17747324 and rs7903146, which were allocated in the HR and LR groups, respectively (table 4). Meanwhile the $\mathrm{P}$-score detected 11 SNPs (including the 2 non-NA SNPs from the M-score) with $-2 \log _{10}\left(A B F_{01}\right)=11.5>10$ (table 5). Both scores detected the 2 most significant SNPs from single-SNP analysis. It is to be noted that ABF values across scores are not comparable, since the 2 scores can give different $\mathrm{ABF}$ values even if the same allocation is used. As per ABF, both allocations from BPM indicated a very strong association of the selected non-NA SNPs with the disease. The high ABFs from both scores seemed to be driven by the very strong association through rs17747324 and rs7903146. Also, the P-score detecting more SNPs than the M-score suggested a possible interaction among the selected SNPs. As can be seen in tables 4 and 5, BPM 
Table 5. The final allocation of the non-null SNPs selected by using a cutoff of 0.4 on the posterior probability of each SNP to be in the non-NA $(\mathrm{LR}+\mathrm{HR})$ group from a BPM P-score analysis in the TCF7L2 gene

\begin{tabular}{|c|c|c|c|c|}
\hline & \multirow{2}{*}{$\begin{array}{l}\text { Posterior } \\
\mathcal{A}\end{array}$} & \multirow{2}{*}{$\begin{array}{l}\text { Posterior } \\
\text { probability }\end{array}$} & \multicolumn{2}{|l|}{ Single-SNP } \\
\hline & & & coefficients & $\mathrm{p}$ values \\
\hline rs7079711 & LR & 0.49 & -0.33 & $5.2 \times 10^{-4}$ \\
\hline rs11196181 & LR & 0.46 & -0.24 & $4.2 \times 10^{-2}$ \\
\hline rs17747324 & HR & 0.61 & 0.42 & $3.6 \times 10^{-11}$ \\
\hline rs7903146 & LR & 0.41 & -0.39 & $1.7 \times 10^{-11}$ \\
\hline rs7079673 & LR & 0.37 & -0.12 & $2.8 \times 10^{-1}$ \\
\hline rs11196228 & LR & 0.27 & -0.33 & $3.1 \times 10^{-2}$ \\
\hline rs7084875 & LR & 0.40 & -0.01 & $8.8 \times 10^{-1}$ \\
\hline rs290483 & $\mathrm{HR}$ & 0.36 & -0.02 & $7.3 \times 10^{-1}$ \\
\hline rs7922641 & $\mathrm{HR}$ & 0.26 & 0.001 & $9.9 \times 10^{-1}$ \\
\hline rs4918801 & HR & 0.52 & 0.11 & $3.7 \times 10^{-1}$ \\
\hline rs10885424 & LR & 0.23 & 0.01 & $9.2 \times 10^{-1}$ \\
\hline
\end{tabular}

The single-SNP results of these non-null SNPs are also listed for comparison. The direction of the single-SNP coefficient and the group allocation by BPM match.

captured some novel SNPs, which again emphasizes the power gain by joint modeling of SNPs within a gene over single-SNP association analysis.

\section{Discussion}

Our BPM approach makes use of the fact that we are interested in the detection of the associated SNPs and not in the estimation of individual SNP effects. The main advantage of classifying the SNPs into these 3 groups is that, for each specific choice of allocation of risk labels to the SNPs, we can model the joint effect of the SNP set on the disease with only 3 parameters. This approach could be especially advantageous when we are considering joint modeling of a large group of SNPs with a relatively small sample size. In addition to this, our proposed approach provides the flexibility of assigning scores to each of these LR or HR groups of SNPs in order to capture the highorder interaction among the SNPs. Our model provides the flexibility of adjusting for other covariate effects (online suppl. material section 9) and allows for modeling of epistatic and nonlinear SNP effects. Here, we considered a pairwise scoring scheme that captures such higher-order interaction among the SNPs. Other scores such as Gaussian kernels can be used to capture these epistatic effects. Our simulation studies and real data analysis demonstrated the usefulness of this proposed method to detect SNPs with higher-order interaction. It is to be noted that we only considered multiplicative interaction in our simulation experiments. In general, the concept of interaction is much broader than multiplicative interaction. We intend to study the performance of BPM for a broader class of interactions in the future.

One advantage of the BPM approach is that it models the latent state of association (risk allocation) of the SNPs given the phenotype and genotype data and thus does not get strongly influenced by the LD among the SNPs. We conducted simulations to study the impact of the LD on power of detection of our BPM approach. In general, we found that the approach loses some power when there is a strong correlation (0.9) among the SNPs, but the performance was very similar between SNPs with no LD and SNPs with moderate LD (0.5). One must note that for large numbers of SNPs in very high LD, the autocorrelation plots will show high autocorrelation even for large values of lag and, hence, more RJMCMC iterations will be needed for convergence. In such a scenario, the BPM chain (due to its single-site updating scheme) is likely to get stuck, which will be indicated clearly in the running mean plot of parameter $\alpha$. On the same note, our assumption of independent prior distributions for risk allocations of all SNPs is reasonable, since we model the latent state of association for each SNP. For our future work, we intend to implement some Markovian structure on the prior distribution to model the dependency among the SNPs and investigate if there is any improvement on the power for detection of association.

One big hypothesis for this BPM approach is that it assumes all of the SNPs within each risk group have the same effect sizes. We investigated the performance of our proposed approach through simulation studies when this assumption is violated (Model 5). In our simulation studies, the proposed approach performed quite well compared to BEAM and LKM, even when the SNPs had very different effect sizes.

One limitation of the current version of the BPM approach is that the update of $\mathcal{A}_{j}$ is realized conditionally on $\mathcal{A}_{(-j)}$ for a locus $j=1,2, \ldots, p$. Given that the space being explored is huge, the sampler is not very computationally efficient in exploring the entire model space. BPM was also found to be somewhat sensitive to starting parameter $\beta$ for the real data analysis. We intend to implement Block-Gibbs sampler and simulated annealing strategies for a better exploration of the model space. We have developed a $\mathrm{C}++$ program for the implementation of our BPM approach. Although potentially this approach could be applied to a large set of SNPs, the current algorithm is more suitable for gene-based association analysis. 


\section{Acknowledgments}

This research was partially supported by NIH grants R21DK089351 and R01DA033958, and the Doctoral Dissertation Fellowship of the University of Minnesota Graduate School. We are grateful to the University of Minnesota Supercomputing Institute for partial support of this work. The Atherosclerosis Risk in Communities (ARIC) Study is carried out as a collaborative study sup- ported by National Heart, Lung, and Blood Institute contracts (HHSN268201100005C,HHSN268201100006C,HHSN268201100007C, HHSN268201100008C, HHSN268201100009C, HHSN268201100010C, HHSN268201100011C, and HHSN268201100012C). The authors thank the staff and participants of the ARIC study for their important contributions. We would also like to thank the two referees for a prompt review of our work and for providing us with helpful feedback.

\section{References}

$\nabla_{1}$ Voight BF, Scott LJ, Steinthorsdottir V, et al: Twelve type 2 diabetes susceptibility loci identified through large-scale association analysis. Nat Genet 2010;42:579-589.

2 Wellcome Trust Case Control Consortium: Genome-wide association study of 14,000 cases of seven common diseases and 3,000 shared controls. Nature 2007;447:661-678.

3 Tibshirani R: Regression shrinkage and selection via the lasso. J R Stat Soc Series B Stat Methodol 1996;58:267-288.

-4 Gayán J, González-Pérez A, Bermudo F, et al: A method for detecting epistasis in genomewide studies using case-control multi-locus association analysis. BMC Genomics 2008;9: 360.

5 Province MA, Borecki IB: Gathering the gold dust: methods for assessing the aggregate impact of small effect genes in genomic scans. Pac Symp Biocomput 2008:190-200.

-6 Bush WS, Dudek SM, Ritchie MD: Biofilter: a knowledge-integration system for the multilocus analysis of genome-wide association studies. Pac Symp Biocomput 2009:368-379.

-7 Chen LS, Hutter CM, Potter JD, Liu Y, Prentice RL, Peters U, Hsu L: Insights into colon cancer etiology via a regularized approach to gene set analysis of GWAS data. Am J Hum Genet 2010;88:860-871.

8 Mukhopadhyay I, Feingold E, Weeks DE, Thalamuthu A: Association tests using kernelbased measures of multi-locus genotype similarity between individuals. Genet Epidemiol 2010;34:213-221.

-9 Pan W: A unified framework for detecting genetic association with multiple SNPs in a candidate gene or region: contrasting genotype scores and LD patterns between cases and controls. Hum Hered 2010;69:1-13.

10 Wu MC, Kraft P, Epstein MP, Taylor DM, Chanock SJ, Hunter DJ, Lin X: Powerful SNPset analysis for case-control genome-wide association studies. Am J Hum Genet 2010;86: 929-942.

11 Larson NB, Schaid DJ: A kernel regression approach to gene-gene interaction detection for case-control studies. Genet Epidemiol 2013; 37:695-703.

-12 Ma L, Clark AG, Keinan A: Gene-based testing of interactions in association studies of quantitative traits. PLoS Genet 2013;9: e1003321.
13 Servin B, Stephens M: Imputation-based analysis of association studies: candidate genes and quantitative traits. PLoS Genet 2007;3: e114.

14 Park MY, Hastie T: Penalized logistic regression for detecting gene interactions. Biostatistics 2008;9:30-50.

15 Guan Y, Stephens M: Bayesian variable selection regression for genome-wide association studies. Ann Appl Stat 2011;5:1780-1815.

16 Li J, Das K, Fu G, Li R, Wu R: The Bayesian lasso for genome-wide association studies. Bioinformatics 2011;27:516-523.

-17 Merryweather-Clarke T, Cadet E, Bomford A, Capron D, et al: Digenic inheritance of mutations in HAMP and HFE results in different types of haemochromatosis. Hum Mol Genet 2003;12:2241-2247.

18 Lunetta KL, Hayward LB, Segal J, Van Eerdewegh P: Screening large-scale association study data: exploiting interactions using random forests. BMC Genet 2004;5:32.

19 Schwartz DF, Ziegler A, König IR: Beyond the results of genome-wide association studies. Genet Epidemiol 2008;32:671.

20 McKinney A, Crowe JE, Guo J, Tian D: Capturing the spectrum of interaction effects in genetic association studies by simulated evaporative cooling network analysis. PLoS Genet 2009;5:e1000432.

21 Conti V, Gauderman JW: SNPs, haplotypes, and model selection in a candidate gene region: the SIMPle analysis for multilocus data. Genet Epidemiol 2004;27:429-441.

22 Lunn J, Whittaker JC, Best N: A Bayesian toolkit for genetic association studies. Genet Epidemiol 2006;30:231-247.

23 Zhang Y, Liu J: Bayesian inference of epistatic interactions in case-control studies. Nat Genet 2007;39:1167-1173.

24 Wakefield J, De Vocht F, Hung RJ: Bayesian mixture modeling of gene-environment and gene-gene interactions. Genet Epidemiol 2010;34:16-25.

25 Fridley BL: Bayesian variable and model selection methods for genetic association studies. Genet Epidemiol 2009;33:27-37.

-26 Marttinen P, Corander J: Efficient bayesian approach for multilocus association mapping including gene-gene interactions. BMC Bioinformatics 2010;11:443.
7 Papathomas M, Molitor J, Hoggart C, Hastie D, Richardson S: Exploring data from genetic association studies using Bayesian variable selection and the Dirichlet process: application to searching for gene $\times$ gene patterns. Genet Epidemiol 2012;36:663-674.

28 Zhang Y, Zhang J, Liu JS: Block-based Bayesian epistasis association mapping with application to WTCCC type 1 diabetes data. Ann Appl Stat 2011;5:2052-2077.

29 Zhang Y: A novel Bayesian graphical model for genome-wide multi-SNP association mapping. Genet Epidemiol 2011;36:36-37.

30 Basu S, Stephens M, Pankow JS, Thompson EA: A likelihood-based trait-model-free approach for linkage detection of binary trait. Biometrics 2010;66:205-213.

-31 Basu S, Pan W, Oetting W: A dimension reduction approach for modeling multi-locus interaction in case-control studies. Hum Hered 2011;71:234-245.

- 32 Sisson SA: Transdimensional Markov chains: a decade of progress and future perspectives. J Am Stat Assoc 2005;100:1077-1089.

-33 Gelman A, Rubin DB: Inference from iterative simulation using multiple sequences (with discussion). Stat Sci 1992;7:457-511.

34 Morris AP, Voight BF, Teslovich TM, et al: Large-scale association analysis provides insights into the genetic architecture and pathophysiology of type 2 diabetes. Nat Genet 2012; 44:981-990.

35 The Atherosclerosis Risk in Communities (ARIC) Study: design and objectives. The ARIC Investigators. Am J Epidemiol 1989; 129:687-702.

-36 Purcell S, Neale B, Todd-Brown K, Thomas L, et al: Plink: a tool set for whole-genome association and population-based linkage analyses. Am J Hum Genet 2007;81:559-575.

37 Liu JZ, McRae AF, Nyholt DR, et al: A versatile gene-based test for genome-wide association studies. Am J Hum Genet 2010;87:139-145.

38 Heidelberger P, Welch PD: Simulation run length control in the presence of an initial transient. Oper Res 1983;31:1109-1144.

39 Wakefield J: Bayes factor for genome-wide association studies: comparison with $\mathrm{p}$ values. Genet Epidemiol 2008;33:79-86.

40 Zheng G, Yang Y, Zhu X, Elston RC: Analysis of Genetic Association Studies, ed 1. New York, Springer, 2012. 